\title{
Neue Leistungen im BEMA: Adhäsivbrücken
}

\author{
Die zahnmedizinische Forschung geht rasant voran. Sowohl im konservierenden Bereich als auch \\ im prothetischen Bereich gibt es stets Neuerungen. Damit solche neuen Leistungen in den Katalog \\ der Kassenleistungen aufgenommen werden, muss sich der Gemeinsame Bundesausschuss (G-BA) \\ damit auseinandersetzen, ob diese Leistungen von der Versichertengemeinschaft übernommen \\ werden. Als solche zu übernehmende Leistung sieht der G-BA jetzt die adhäsiven Brücken in neuer \\ Form an. Der Bewertungsausschuss hat eine neue Leistungsbewertung vorgenommen.
}

Bis vor Kurzem war die Adhäsivbrücke unter der BEMA-Nr. 93 abzurechnen und mit 335 Punkten bewertet. Bei Einhaltung der Vorgaben konnten adhäsiv befestigte einspannige Brücken im Frontzahnbereich mit Metallgerüst als Regelversorgung, aber nur bei Versicherten im Alter zwischen 14 und 20 Jahren, erbracht und abgerechnet werden.

Die BEMA-Nr. 93 wurde ab dem 1. Juli 2016 durch zwei neue Gebührennummern und weniger Einschränkungen ersetzt:

- BEMA-Nr. 93a: Adhäsivbrücken mit Metallgerüst im Frontzahnbereich mit einem Flügel

- BEMA-Nr. 93b: Adhäsivbrücken mit Metallgerüst im Frontzahnbereich mit zwei Flügeln

Mit der zweiflügeligen Brücke ist die alte BEMA-Nr. 93 beschrieben, so dass die BEMA-Nr. 93b ebenfalls mit 335 Punkten bewertet ist. Die geringere Bewertung der BEMA-Nr. 93a mit 240 Punkten berücksichtigt den reduzierten Zeitaufwand bei der einflügeligen Brücke. Die neuen Gebührennummern können nur abgerechnet werden für Adhäsivbrücken, die dem Ersatz von Schneidezähnen dienen. Insbesondere können also Eckzähne nicht ersetzt werden. Die vormalige Altersbegrenzung wurde aufgehoben, so dass die Versorgung grundsätzlich für alle
Versicherten unabhängig ihres Alters in Betracht kommt. Eine Einschränkung besteht nur beim Ersatz von zwei nebeneinander fehlenden Schneidezähnen: Hier kann weiterhin eine Abrechnung nach BEMA nur bei Versicherten erfolgen, die das 14 . aber noch nicht das 21. Lebensjahr vollendet haben.

Moderne Möglichkeiten wie Fräsungen von Zirkonoxid und moderne Adhäsivverfahren lassen hier viele Möglichkeiten für ästhetisch sehr anspruchsvolle Lösungen zu. Wie und unter welchen besonderen Kriterien außervertragliche Klebebrücken berechnet werden, zeigt unser Beitrag von Dr. Dr. Alexander Raff zu den Adhäsivbrücken nach den GOZ-Nrn. 5150 und 5160.

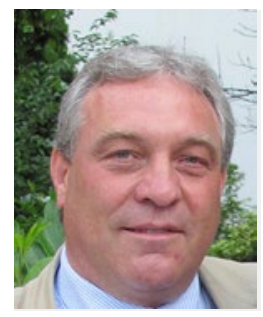

Dr. Christian Öttl

Referent Honorierungswesen des Bundesvorstandes des FVDZ

\section{Ergänzungsleistungen - Der gangbare Weg}

\section{Adhäsivbrücken - alles andere als einfach}

\begin{abstract}
Hinter der Formulierung der "Versorgung eines Lückengebisses mit Hilfe einer durch Adhäsivtechnik befestigten Brücke" in der Leistungslegende der GOZ-Nummer 5150 verbergen sich die Klebebrücken (Synonyme: Marylandbrücken oder Adhäsivbrücken), die nur nach der zu überbrückenden Spanne berechnet werden: die GOZ-Nummer 5150 für die erste Spanne, die um mehr als 50 Prozent geringer bewertete GOZ-Nummer 5160 für jede weitere zu überbrückende Spanne. Klebebrücken nach der GOZ sollten aus diversen Gründen mit besonderem Augenmerk bedacht beziehungsweise suffizient bewertet werden, da auch die Berechnung dieser prothetischen Variante alles andere als einfach ist.
\end{abstract}

Wie im vorausgehenden Beitrag „Neue Leistungen im BEMA: Adhäsivbrücken“ aufgeführt, gibt es seit einem Jahr im BEMA die Unterscheidung von Adhäsivbrücken mit einem Flügel (BEMA-Nummer 93a) und mit zwei Flügeln (BEMA-Nummer 93b). Eine derar- tige Unterscheidung existiert in der Gebührenordnung für Zahnärzte (GOZ) von 2012 nicht, die sich bei der Berechnung explizit nur an der Spanne (oder an Spannen) orientiert, nicht aber an der Anzahl der Flügel beziehungsweise der Befestigungsanker. 